\title{
Localisation of corneal foreign bodies
}

\author{
L G Kaye-Wilson
}

\begin{abstract}
Of 50 patients attending the eye casualty department with a corneal foreign body (FB), 41 were able to say where they felt the FB and $78 \%$ of these were localised correctly for side or level of cornea. Patient handedness did not influence FB location. Indicating the upper lid was a particularly poor guide to localisation, whereas FB sensations within the palpebral fissure, in the lower lid or medially or laterally were good guides to actual FB location. A simple method of recording FB location by zone and clock hour is proposed.

(Brf Ophthalmol 1992; 76: 741-742)
\end{abstract}

Corneal foreign body (FB) removal constitutes a significant proportion of the workload of both general and ophthalmic casualty departments. General practitioners, industrial nurses, and general casualty officers are involved in corneal FB management. It would be useful if some means of directing their search for the location of a corneal FB existed, especially if a slit-lamp is not available.

Pain from a corneal FB is usually projected to the eyelid. The accuracy of this pain projection as a method of self-localisation of corneal FBs was investigated by developing a simple system to record corneal FB position and comparing this with the patient's self-localisation.

\section{Methods}

Fifty consecutive patients with metallic corneal FBs (and no previous ocular disease) were seen in the ophthalmic casualty department. A note was made of the mode of injury, the patient's occupaStreet, Edinburgh EH tion, which eye was injured, whether the patient was right or left-handed, and how the FB was removed. Localisation was assessed by asking the patient to point to where he felt the discomfort (with the eye open). This was recorded as a

Accepted for publication 22 July 1992
Figure 1 Patient selflocalisation coding.

Schematic right eye (the left is a mirror image). Score $A-E=$ horizontal; score

$1-5=$ vertical.
Princess Alexandra Eye 9 A

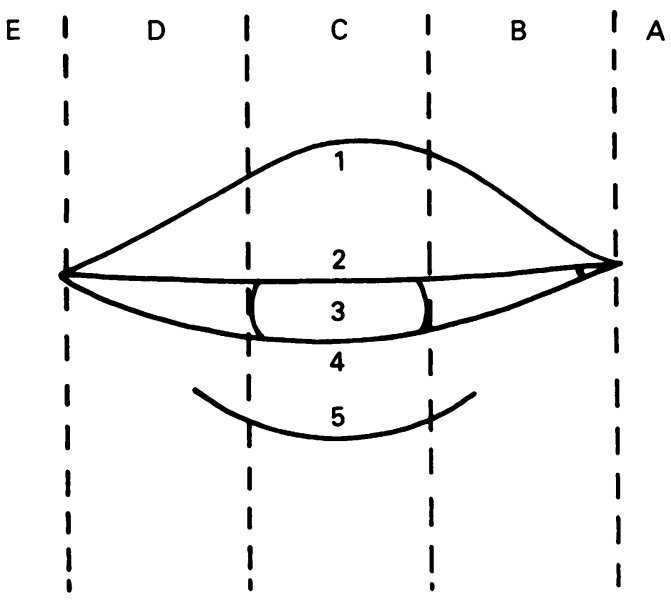

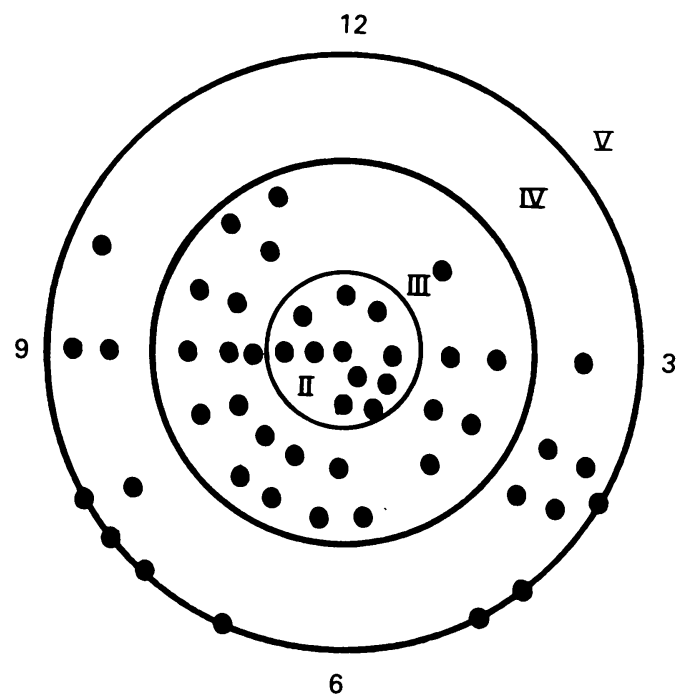

Figure 2 Corneal location coding: zone I central point zone II paracentral third, zone III mid third, zone IV peripheral third, zone $V$ limbal. Fifty corneal $F B$ locations are shown.

vertical (1-5) and horizontal (A-E) localisation score as shown in Figure 1. After slit-lamp examination, FB location was recorded by zone and clock hour (Fig 2), using an abbreviated code. For example, $\mathrm{R} \mathrm{II} / 3^{30}$ denotes the paracentral third of cornea at the half-past-three position in the right eye. FBs were removed under local anaesthesia by cotton bud, 21-gauge needle or, if more adherent or deeper, with a burr. ${ }^{12}$

\section{Results and analysis}

Ninety four percent of patients in this study (47/50) were male; $72 \%(36 / 50)$ of FBs were work-related. Of these patients, $89 \%(32 / 36)$ had not been wearing any eye protection at the time of injury. Twenty eight of $50 \mathrm{FBs}$ could be removed by needle alone; $20 / 50$ required burr removal; only $2 / 50$ were removed by swab alone. There was no significant difference in frequency of needle versus burr FB removal between any of the groups described below.

\section{ACCURACY OF LOCALISATION}

All 50 FB locations are summarised in Figure 2, by zone and the nearest clock hour. Actual FB locations were compared with localisations (where the patients believed the FBs were situated by their sensation of pain). For example, if the FB was located in the upper half of the cornea, vertical localisation was correct if the patient felt the FB in the upper lid (score 1-2 in Fig 1). Similarly, if the FB was located in the medial half of the cornea, horizontal localisation was correct if the patient felt the FB medially (score A-B in Fig 1) 
Nine patients found FB localisation impossible, including three who did not feel the FB. Of the remaining 41 patients who believed they could localise the FB sensation, 14 localised correctly to both side and level, whilst 18/41 localised correctly to side only or level only - that is, $32 / 41(78 \%)$ localised side and/or level correctly. Only $9 / 41$ patients localised incorrectly in both directions and of these, $89 \%(8 / 9)$ were misleadingly felt in the upper lid.

Twenty three patients indicated that they felt the FB in the upper lid (score 1-2): however, only $7 / 23(30 \%)$ actually had an FB in the upper half of the cornea. On the other hand, nine patients felt the FB in the lower lid (score 4-5), and seven of these $(78 \%)$ did have an FB present in the lower half of the cornea. Similarly, nine patients said that they felt the FB within the palpebral aperture (score 3); seven of these (78\%) had an FB on the central horizontal line from 9 o'clock to 3 o'clock, with the other two being just below the line.

Thirty one patients felt pain from the FB either nasally or temporally (score A, B, D, E); 74\% (23/31) were localised correctly with respect to the 12 o'clock to 6 o'clock vertical line, with equal accuracy nasally and temporally.

\section{EFFECT OF HANDEDNESS}

To assess whether patient handedness influenced the position of the FB on the cornea, patients were grouped into those with an FB in the eye of the dominant side $(22 / 50)$ and those with an FB in the eye of the non-dominant side (28/50).

Patients were also divided into those with an FB on the right half or the left half of the cornea, regardless of which eye the FB had landed on. Each cornea was divided into right and left sides by a vertical line from 12 o'clock to 6 o'clock. Seven FBs were recorded on this line. Excluding these, 19 out of 43 landed on the dominant side of the cornea.

\section{Discussion}

As a result of this study it appears likely that handedness does not influence FB location.
Percival described a trend towards injury of the eye of the non-dominant side in his study of intraocular FBs. ${ }^{3}$ However, non-use of eye protection is of very much greater significance.

Most of the FBs landed on cornea within the palpebral aperture as the eyelids protect the remaining cornea. However, without the aid of a slit-lamp, it is often difficult to locate corneal FBs. This study suggests that FBs felt under the upper lid are unlikely to be in the upper half of the cornea, whereas FBs felt in the lower lid are likely to be in the lower half of the cornea. Similarly, if discomfort from an FB is felt within the palpebral aperture and not on the lid margins, the $F B$ is very likely to be on the central band of cornea. Also, nasal or temporal localisation correlates well with actual location.

No universal system of recording corneal FB location is in use. Precise records are clearly of medicolegal importance: for example, multiple corneal scars may be present in a case for an industrial compensation claim, but which scar is relevant to the claim?

There are many alternatives to the system of recording FB location used in this study. Drawing a dot inside a circle to represent a corneal FB is inaccurate. Verbal records are often vague 'central' may in fact be paracentral. Slit-lamp measuring grids and messy 'grid' rubber stamps for the case notes are an added expense. In contrast, the clock hour scheme is easily understood and accurate (although zones are more easily remembered by name than number).

This study has shown a useful clinical application for corneal sensitivity mapping. ${ }^{5}$ It is also hoped that the system of recording corneal FB location might be introduced into general use.

1 Sigurdsson H, Hanna I, Lockwood AJ, Longstaff S. Removal of rust rings, comparing electric drill and hypodermic needle. Eye 1987; 1: 430-2.

2 Brown N, Clemett R, Grey R. Corneal rust removal by electric drill. Clinical trial by comparison with manual removal. drill. Clinical trial by comparis

3 Percival SBP. A decade of intraocular foreign bodies. Brf Ophthalmol 1972; 56: 454-61.

4 Liu C, Davison C, Cooling R. Eye protection in the metal working industry. $\mathrm{BrMed} \mathcal{F}$ 1990; 301: 1048.

5 Boberg-Ans J. Experience in clinical examination of corneal sensitivity. Br f Ophthalmol 1955; 39: 705-26. 